\title{
Retraction Note to: Identification of core miRNA based on small RNA-seq and RNA-seq for colorectal cancer by bioinformatics
}

\author{
Youwei Kou $^{1} \cdot$ Lei Qiao $^{2} \cdot$ Qiang Wang ${ }^{1}$
}

Published online: 17 August 2015

(C) International Society of Oncology and BioMarkers (ISOBM) 2015

Retraction Note to: Tumor Biol. (2015) 36:2249-2255

DOI 10.1007/s13277-014-2832-x

The Publisher and Editor retract this article in accordance with the recommendations of the Committee on Publication Ethics (COPE). After a thorough investigation we have strong reason to believe that the peer review process was compromised.

The online version of the original article can be found at http://dx.doi.org/ 10.1007/s13277-014-2832-x.

Qiang Wang

wangq@sj-hospital.org

1 Department of Gastrointestinal and Nutriology Surgery, Shengjing,

Hospital of China Medical University, Sanhao Street No. 36,

Shenyang 110004, People's Republic of China

2 Department of Colorectal Surgery and Oncology, Shenging Hospital of China Medical University, Sanhao Street No. 36,

Shenyang 110004, People's Republic of China 